\title{
Research on the Teaching Experiment Management System Based on JSP
}

\author{
Liu Yang \\ Chongqing College of Electronic Engineering,Chongqing,China
}

173676096@qq.com

Keywords: Teaching Experiment Management System, JSP

\begin{abstract}
With the deepening reform of higher education, teaching experiments must adapt teaching reform situation, intensify the reform of teaching content and methods of reasonable use of modern information technology to build powerful, rational structure of the teaching experiment management system, it is an important measure to promote the teaching experiment reform This paper introduces the key issues a web-based teaching experiment management system design and implementation.
\end{abstract}

\section{Introduction}

Under Credit, requires experimental teaching students to give full freedom of time and content, teaching laboratory must comply with the requirements of this open teaching. Open teaching experiment in teaching management first face teaching target, content, time and other factors of uncertainty, coupled with individualized teaching plans, so that increase the difficulty of management, and because of individualized education leads to an increase in management and teaching clue, duplication increases, resulting in management and teaching workload increased several times even to the number of times. Thus, although the majority of teachers and laboratory staff recognize the importance and necessity of open laboratory, but in the face of open experimental teaching workload may bring increase and complicate the management idea generally reluctant to open experimental teaching. Therefore, we must experiment to the traditional way of teaching management reform, full use of modern information technology to explore more effective teaching experiment management, development of web-based teaching experiment management system to improve the level of management, teaching experimental reform to provide technical support.

\section{Design Goals Analysis of Teaching Experiment Management System Based on JSP}

Teaching experiment management system to optimize management processes, improve management efficiency for the purpose, to achieve the goal the following aspects.

To Achieve Individualized Teaching Management. Utilization of Network Information technology teaching plans and personalized management of the teaching process. On the one hand to achieve individualized teaching plan management, pilot projects that can be specified in the student teaching program is selected, until not less than the required basic science scores are each student achieve a teaching program. on the other hand the teaching process of a network pilot project appointments the way, students can query through the network teaching experiment, the freedom of the selected time, appointment experimental project, to ensure that students choose the content of the freedom of the experiment time.

Improve the Hierarchical Management Efficiency of Teaching Department, Teaching Laboratory. According to the school, the hospital (department), laboratory managers concerned corresponding duties were set different operating authority data through all levels of management network management platform for real-time and non-real-time interaction, the traditional manual management to paperless management information .113 reduce the administrative workload of teaching experiment using a database management program teaching students basic information, 
booking experiment results, the completion of the experiment, the workload of teachers and other information, the major teaching statistics, analysis done by the computer maximize reduce the workload of teaching experiment management.

\section{The Advantages of JSP}

Write Once Run Anywhere. JSP technology inherited innate JAVA Advanced Concepts - "Write Once Run Anywhere". This concept is increasingly far-reaching impact on the Internet industry, interactive Web pages design. JSP pages can be easily cross-platform, cross-web server software to design and develop the source code.

Web server in the event of a request to access JSP page, the first implementation of the program fragment, then the implementation of the results returned to the client in HTML format. Program fragments can operate the database, redirect pages and send E-mail, etc., which is to establish a functional dynamic websites require. All program operations are executed on the server, network upload to the client's only the result obtained on the client browser requirements are minimal.

Support A Variety of Dynamic Web Page Format. Currently using JSP technology designed pages should take what kind of format do not have a clear standard. In general, JSP technology is currently only supports HTML / DHTML traditional browser file format, and can be applied to support wireless communication devices such as mobile phones, PDA and other devices for web browsing WML file format, it can also support other B2B applications the XML format.

Although the current JSP engine does not require the generation of XML representation, but JRun Servlet engine can create an XML representation in the series JSP pages and generate Java source code simultaneously. So we can see the future as a JSP XML generator look. Because XML will play an important role in the future of Web applications, such as wireless access protocol (WAP) has been used as a digital phone pocket organizer networking standard, is based on the use of XML content conversion [4] Sun Company believes.

\section{The Features of JSP}

Compared with other conventional WEB application technology, JSP technology has its very distinctive features:

JSP Tag Scalability. Although ASP and JSP are using tags and scripting techniques to create dynamic WEB pages, JSP technology enables developers to extend JSP tags to be applied, JSP developers can custom tag libraries, so web authors take full advantage of XML-compliant tag technology powerful function, greatly reducing reliance on scripting language. Since custom tag technology that enables authors to reduce the extent and complexity of making web pages more pages to expand critical functions.

JSP Cross-Platform Reusability. JSP developers in the development process has been concerned reusability. JSP components (Enterprise JavaBeans, or custom JSP tags) are reusable across platforms. Enterprise JavaBeans components can access traditional database, and are able to work in a distributed mode system UNIX and WINDOWS platforms. JSP technology provides an easy tab for the extension developers, XML-compatible interface that is shared pages packed full of features that make industrial standardization.

Ease of Maintenance. JSP technology-based applications easier to maintain and manage than ASP-based applications. Scripting languages are well served in small applications, but can not adapt to large, complex applications. Because, JAVA is structured, it is relatively easy to create and maintain a large component of the application. JSP prominent component technology allows content without affecting the logic or modified without affecting the contents of the logic becomes very easy to implement. Javabeans structure of the enterprise-level integrated business logic, such as database access, security, transaction integrity.

Diversity of Enterprise Product. JAVA2 Platform as Enterprise Edition (J2EE) is applied to the structure of the enterprise JAVA applications, as part of J2EE, JSP pages can access all J2EE components, including JavaBeans, and Enterprise JavaBeans and JAVAServlets. JSP pages can be 
fully compiled into Servlets, they are entitled to the characteristics and flexibility for server-side JAVA applications.

\section{Dynamic Web Design Based on JSP}

JSP's built-in scripting language is based on the Java programming language, and Java is a software programming language designed for Internet development leaving the web page (Web Page) Switch to the static and dynamic, and can produce "applet (Applets)" a simplified language. It has hampered the spread of a computer virus functionality; Java client machines to break the language environment and structure of the CPU, you can do "write once, run everywhere (the Write Once, Run Anywhere)". The emergence of Java JSP formal step toward a bright stage web technologies and applications. You can use it to write a cross-platform technology running everywhere, the versatility of Java, JSP ease of use, Servlet combines the speed, the use of platform deployment tips on effectively shield the Java question of pace, it's Web design has a strong technological advantage.

To generate and display the contents of the separation using JSP technology, Web page developers can use HTML or XML logo to design and format the final page. Use JSP logo or small script to generate dynamic content on the page. Generated content logic is encapsulated in the logo and JavaBeans components and tied in small script, all the scripts run on the server side. If the core logic is encapsulated in the logo and Beans, then others, such as management and Web page designers, can edit and use the JSP page without affecting the content generated.

On the server side, JSP engine interprets the contents of JSP logo and small scripts, generated requested (for example, by accessing JavaBeans components, using JDBC technology to access the database, or include file), and the result is transmitted in the form of HTML (or XML) page back to the browser. This helps authors to protect their code, but ensure that any HTML-based Web browser, full availability.

Most JSP pages rely on reusable, cross-platform components (JavaBeans or EnterpriseJavaBeans components) to perform more complex processing application requires. Developers can share and exchange components to perform common operations, or to make these components more users or groups of customers are using. Based on the component to accelerate the overall development process, and makes all kinds of organizations have been balanced in their existing skills and optimize the results of development efforts.

Web page developers are not familiar with the scripting language programmers. JSP technology encapsulates many features, these features are required for dynamic content generation in use, XML and JSP-related identity of. JSP standard logo to access and instantiate JavaBeans components, set or retrieve the component properties, use other methods to download Applet, and the implementation of more difficult to coding and time-consuming function.

By developing custom tag libraries, JSP technology is extensible. In the future, third-party developers and other personnel can create their own logo library frequently used functions. This allows Web page developers can use familiar tools and the same logo as the member to perform specific functions to work.

JSP + JavaBean + Servlet three-tier structure of the substance is more of a Controller: Servlet to distribute the client browser requests. If the controller plays the role of the Servlet role in understanding the client's request for the pretreatment of Servlet understanding will be of great help. Web.xml configuration file can be found by user requests and correspondence between a specific Servlet, each Servlet Servlet has a specific corresponding object, so that the handle is a user requests a Servlet objects inherited from HttpServlet.

\section{The Functions Configuration of Experiments and Teaching Management System Based on JSP}

Student System. Students in the laboratory after selected and successfully logged in, students at this interface can do the following: (1) submit the plan: Students must first select pilot projects as 
their own teaching programs (2) appointment experiment: Students can easily Select an appointment time to do a test, but must answer the question raised by the experiment (all questions and answers are randomly arranged), the only book the experiment (3) information query: You can view the experiment booking situation, performance, etc. Teaching situation (4) Change password: students can modify their passwords into any of not more than 16 letters or numbers from a password, students can information security be guaranteed.

Teachers System. After the teachers to their own identity and their own password can be the appropriate permissions to work, the main function as follows: (1) Project Management: Laboratory experiments approved project name, modify, increase or decrease the experimental project; to set each item accommodate a maximum of the number of students, etc. (2) information management: announcement, respond to student questions (3) the reservation management: the management of the reservation program, students locked reservation information (4) teacher management: laboratory director can add, delete and teachers Related information (5) student management: students can participate in the experiment attendance, may also increase or delete the list of students, etc. (6) performance management: after the experimental class teacher to student assessment test is completed to sign student pilot project on the Internet results (7) statistical query: query, statistics of all relevant information [1].

Educational Administration System. Teaching experiment management input identity and password, after verification by access to educational management system interface, educational administration staff here can do the following: (1) the task of teaching: teaching load assigned to each laboratory, each validation of Experimental Program (2) laboratory management: modify or add the name of the laboratory experiment in the system and initializes the experiment (3) information management: responsible for Home announcement, delete, send and receive electronic information between laboratories, and to view and respond to student feedback . the information, etc. (4) statistical inquiry: inquiry, statistics, or any school laboratory teaching situation; in charge of school leadership, the Senate has (4) rights institution (department) can only view the Senate Court (Department) teaching experimental conditions ,

\section{Conclusions}

Experimental teaching system to achieve a computer network management, students are free to choose the pilot project and experimental time through the network, both to achieve the goal of individualized teaching management, but also greatly reduce the workload of teachers in teaching management, improve the teaching experiment management efficiency, better way to solve the problem of opening laboratory management, and promote the process of reform and opening laboratory, and we design a system to take full account of the openness of the system, and the use of mainstream technology, we will further strengthen and further optimize the system , enhanced compatibility, security, and even the proposed increase in student campuses across laboratory experiments elective project management functions, and prepared to carry out supporting network-assisted teaching system, full use of multimedia and virtual reality technology to improve the quality of teaching experiment, make the system teaching experiment reform play a greater role.

\section{References}

[1] Yu Dahong: Laboratory Research and Exploration, Vol. 6 (2004) No 53, p.25-26

[2] Liu Jinde: Computer Application, Vol. 12 (2005) No 27, p.74-76

[3] Liao Jun: Computer Application, Vol. 1 (2006) No 33, p.11-14

[4] Miao Chunyu: Communications, Vol. 3 (2007) No33, p.121-124

[5] Hu Guangqiang: Laboratory Research and Exploration, Vol. 1 (2006) No 33, p.11-14 